Check for updates

Cite this: RSC Adv., 2020, 10, 16987

\section{Dietary milk fat globule membrane supplementation during late gestation increased the growth of neonatal piglets by improving their plasma parameters, intestinal barriers, and fecal microbiota $\uparrow$}

Xiangyu Zhang, ${ }^{a}$ Yujun Wu, ${ }^{a} \mathrm{Hao}$ Ye, ${ }^{a}$ Cuiping Feng, ${ }^{\mathrm{b}}$ Dandan Han, ${ }^{\mathrm{a}}$ Shiyu Tao, ${ }^{a} \mathrm{Yu} \mathrm{Pi}{ }^{\mathrm{a}}$ Junying Zhao, ${ }^{c}$ Lijun Chen ${ }^{c}$ and Junjun Wang $\mathbb{D}^{\text {*a }}$

\begin{abstract}
Milk fat globule membrane (MFGM), mainly comprising protein and fat, has been reported to have multiple biological functions for relieving intestinal inflammation and enhancing growth performance. We hypothesized that MFGM supplementation into sows' diet during late gestation can have a positive impact on the intestinal microecology of sows and their piglets, as well as on the growth of neonates. Therefore, the present study was conducted to investigate such effects and their potential mechanisms. Twenty-two pregnant sows were selected and randomly divided into the CON group (basal diet) and MFGM group (basal diet supplemented with $9.9 \mathrm{~g}$ per day MFGM). The feeding period began from d 85 of gestation and until farrowing. The concentrations of albumin (ALB), low-density lipoprotein cholesterol (LDL-C), and non-esterified fatty acids (NEFA) in plasma and short-chain fatty acids (SCFAs) in feces of sows from the MFGM group were determined. The concentrations of growth hormone (GH), immunoglobulin A (IgA), glucose (GLU), and NEFA in the umbilical cord blood in the MFGM group were significantly higher than those in the CON group. Piglets from the MFGM group showed improved growth performance, increased villus height in the jejunum, decreased crypt depth in the duodenum and jejunum, upregulated mRNA expressions of tight junctions (namely, Occludin; Claudin-1, Claudin-2, and Claudin-4; zonulin-1 (ZO-1)); mucins such as Mucin 2, Mucin 4, Mucin 13, and Mucin 20; and immunerelated genes, such as tumor necrosis factor- $\alpha$ (TNF- $\alpha$ ), interferon- $\gamma$ (INF- $\gamma$ ), interleukin-22 (IL-22), tolllike receptor 2 (TLR2), and toll-like receptor 4 (TLR4). In addition, the abundance of Prevotella in the feces of sows at farrowing and the abundance of Christensenellaceae_R-7_group in the feces of 21 dayold piglets from the MFGM group were significantly higher. Further correlation analysis revealed that the Christensenellaceae_R-7_group was positively correlated with the relative mRNA expressions of Occludin, Claudin-1, Claudin-2, Claudin-4, Mucin 13, TLR2, and TLR4. In conclusion, MFGM supplementation during late gestation improved the physiological status of sows by improving their plasma parameters and intestinal microecology. The improved provision of nutrients through the umbilical cord blood and optimized microbiota colonization in neonatal piglets were beneficial to the intestinal morphological structure and barrier functions, consequently improving the growth performance of neonates during lactation. These findings provide insights into the future applications of MFGM with regard to maternal-fetal nutrition and a new option for nutritional intervention of neonates through maternal dietary manipulation.
\end{abstract}

Received 21st March 2020 Accepted 8th April 2020

DOI: $10.1039 / \mathrm{dOra02618b}$

rsc.li/rsc-advances

\section{Introduction}

${ }^{a}$ State Key Laboratory of Animal Nutrition, College of Animal Science and Technology, China Agricultural University, Beijing 100193, China.E-mail: wangjj@cau.edu.cn ${ }^{b}$ Department of Obstetrics and Gynecology, China-Japan Friendship Hospital, Beijing 100029, China

${ }^{c}$ National Engineering Center of Dairy for Early Life Health, Beijing Sanyuan Foods Co. Ltd, Beijing 100163, China

$\dagger$ Electronic supplementary information (ESI) available. See DOI: 10.1039/d0ra02618b
Late gestation is a critical period since two-thirds of the fetal growth occurs during the last third of the gestation period, which places an intensive physiological and metabolic burden on mothers. ${ }^{1}$ Insufficient or unbalanced nutrition provision for mothers can not only affect maternal health, but also determine the growth and development of fetuses and neonates, as well as induce the risk of metabolic syndrome in their offspring. ${ }^{2}$ 
Therefore, nutritional supply during late gestation is important, considering its direct effects on the reproductive performance of mothers and also having great significance toward fetuses and neonates.

Intestinal development, particularly of the mucosal epithelium, plays an important role in piglet health. ${ }^{3,4}$ Incomplete intestinal function can lead to decreased digestion, absorption, barrier, and immunity. ${ }^{5}$ On the other hand, increased attention has been recently paid toward the role of the microbiota in intestinal development. ${ }^{6}$ Some studies have shown the correlations between the microbiota and intestinal barrier functions. ${ }^{7-9}$ Considering that the microbiota of piglets is mainly derived from the intestinal strains of sows, ${ }^{\mathbf{1 0}}$ regulating the maternal intestinal microecology deserves attention.

Milk fat is one of the main components of milk, generally accounting for $3-5 \%$ of the milk composition in the form of fat balls. On the surface of milk fat, a 10-20 nm membrane is present, which is known as the milk fat globule membrane (MFGM), ${ }^{\mathbf{1 1}, 12}$ which is composed of a three-layer membrane structure comprising phospholipids, sphingolipids, and various proteins. MFGM has been confirmed to be associated with many physiological functions, ${ }^{\mathbf{1 3}}$ such as decreasing infections and inflammations in rodent models, ${ }^{\mathbf{1 4}}$ promoting gut mucosal integrity during lipopolysaccharide (LPS)-induced intestine inflammation in male BALB/c adult mice, ${ }^{15}$ and alleviating high-fat-diet-induced colon inflammation. ${ }^{16}$ MFGM supplementation has also been demonstrated to improve the growth performance of neonatal mice during their early life. ${ }^{17}$ However, there are no studies investigating the effects of MFGM supplementation in the gestational diet on the intestinal microecology of their offspring.

We hypothesized that gestational supplementation with MFGM can have a positive impact on the intestinal microecology of sows and consequently their piglets. Subsequently, this study was conducted to investigate the effects and potential mechanisms of dietary MFGM supplementation during late gestation on litter performance of the sows and also on the gut microbiota, intestinal barrier functions, plasma parameters, and growth performance of their piglets during the neonatal stage.

\section{Materials and methods}

\section{Animals and experimental treatments}

In this study, 22 Large White and Landrace sows (parity: $1.68 \pm$ $0.46)$ were selected and randomly divided into 2 groups: CON group (basal diet) $(n=11)$ and MFGM group (basal diet supplemented with $9.9 \mathrm{~g}$ per day MFGM) $(n=11)$. The formula of the basal diet is shown in ESI Table S1.† The composition of MFGM (Hilmar, USA) is illustrated in ESI Table S2. $\dagger$ Here, $3.3 \mathrm{~kg}$ MFGM was supplemented into the basal diet per ton. Each sow was fed with a $3 \mathrm{~kg}$ diet every day. The feeding period began from d 85 of gestation and continued until farrowing. All the sows were housed in individual gestation stalls $\left(2.1 \times 0.6 \mathrm{~m}^{2}\right)$ from d 85 until d 106 of gestation. On d 107 of gestation, the sows were moved into the farrowing rooms with environmentcontrolled systems and housed in individual farrowing crates $\left(2.1 \times 1.5 \mathrm{~m}^{2}\right)$. Room temperature was maintained at $21.6{ }^{\circ} \mathrm{C}$. All the animal procedures were performed in accordance with the Guidelines for Care and Use of Laboratory Animals of China Agricultural University and approved by the Animal Ethics Committee of China Agricultural University.

\section{Measurement of litter performance and growth performance}

The number of total piglets, live piglets, healthy piglets, weak piglets (birth weight: $<1.0 \mathrm{~kg}$ ), and stillborn fetuses per litter were recorded at birth. The total litter weight, average birth weight, and variation in birth weight were measured and calculated. Piglets per litter were weighed on d 0, d 7, d 14, and d 21 after birth; further, the average body weight and average daily weight gain were calculated for each group.

\section{Sample collection}

Sow. Fresh feces samples as well as blood samples from the ear vein of each sow on d 114 of gestation and umbilical blood at farrowing were collected without fasting. Heparin sodium was used for anticoagulation.

Piglet. Five piglets were randomly selected from each group. On d 21 after birth, the feces samples were collected without fasting; then, all the selected piglets were humanely euthanized for collecting the samples, namely, the middle segments of the duodenum, jejunum, and ileum and the chyme and mucosa from the middle segments of the ileum and colon. The middle segments of the ileum and colon in the piglets were opened and thoroughly rinsed with sterile normal saline; then, the mucosa was collected by scraping with glass slides. Mid-ileal and midcolonic chyme were collected in sterile tubes.

All the blood samples were centrifuged at $3000 \times g$ for $10 \mathrm{~min}$ to obtain the plasma samples and immediately stored at $-20{ }^{\circ} \mathrm{C}$ until further analysis. All the feces samples and tissue samples were stored in liquid nitrogen.

\section{Measurement of plasma parameters}

All the plasma samples were thawed and completely mixed before analysis. The concentrations of biochemical parameters, such as total protein (TP), albumin (ALB), globulin (GLB), creatine kinase (CK), serum total cholesterol (TC), total triglyceride (TG), high-density lipoprotein cholesterol (HDL-C), lowdensity lipoprotein cholesterol (LDL-C), very-low-density lipoprotein cholesterol (VLDL-C), blood glucose (GLU), total bile acid (TBA), and non-esterified fatty acid (NEFA) were determined by means of an automatic biochemical analyzer (Hitachi High-Tech Corporation, Japan). The concentrations of growth hormone $(\mathrm{GH})$, insulin-like growth factors (IGF-1), diamine oxidase (DAO), and immunoglobulins (IgA, IgG, and IgM) were measured by means of ELISA assays according to the manufacturer's instructions (Beijing Sino-UK Institute of Biological Technology, China).

\section{Morphological analysis of intestinal epithelial tissues}

Intestinal samples were removed from $10 \%$ phosphate-buffered formalin, dehydrated through a graded ethanol series $(70 \%$ to 
$100 \%$ ), and then cleared with xylene and embedded in paraffin wax. Serial sections (thickness: $5 \mu \mathrm{m}$ ) were cut with a LEICA RM2135 rotary microtome (Leica Microsystems GmbH, Germany) and stained with hematoxylin and eosin. A minimum of 15 intact and well-oriented villis and their associated crypts from each segment were measured at $100 \times$ magnification under the bright field on a Zeiss Axio Imager microscope (Carl Zeiss Microscopy LLC, United States). The villus height was measured from the tip of the villi to the villus crypt junction; the crypt depth was defined as the depth of the invagination between adjacent villi. ${ }^{17}$

\section{RNA isolation, cDNA synthesis, and real-time quantitative PCR (RT-qPCR)}

Following the protocol, the total RNA data from mid-ileum and mid-colon were extracted by using a TRIzol kit (Invitrogen, United States), and cDNA was obtained by using PrimeScript ${ }^{\mathrm{TM}}$ RT kit (Takara, Japan). RT-qPCR was performed according to the SYBR Premix Ex Taq ${ }^{\mathrm{TM}}$ II instructions (Takara, Japan). Primers for RT-qPCR were synthesized by Shanghai Generay Biotech Co., Ltd. (ESI Table S3†). ${ }^{17-21}$ Amplifications were performed on a LightCycler system (Roche, Germany) in triplicate for each sample. The relative mRNA expressions of the target genes to that of glyceraldehyde-3-phosphate dehydrogenase (GADPH) were calculated according to the $2^{-\Delta \Delta \mathrm{Ct}}$ method.

\section{Fecal microbiota analysis}

The fecal samples were extracted for the total genomic DNA by using the QIAamp® Fast DNA Stool Mini Kit (QIAGEN Ltd., Germany) according to the manufacturer's protocol. The V3-V4 region of the $16 \mathrm{~S}$ rRNA gene was amplified using the universal primers 338F (ACTCCTACGGGAGGCAGCAG) and 806R (GGACTACHVGGGTWTCTAAT). ${ }^{22}$ The amplified products were detected by using $2 \%$ agarose gel electrophoresis, purified by using AxyPrep DNA Gel Extraction Kit (Axygen Biosciences, United States), and quantified by a Qubit 2.0 Fluorometer (Thermo Fisher Scientific, United States). The purified PCR products were pooled into equimolar amounts and sequenced on the Illumina HiSeq 2500 platform to generate paired end reads of 300 bp. $^{23}$

Raw paired-end reads were strictly analyzed by using QIIME (version 1.9). ${ }^{23}$ In brief, the low-quality sequences with a length of $<220 \mathrm{nt}$ or $>500 \mathrm{nt}$, average quality score of $<20$, and sequences containing $>3$ nitrogenous bases were removed. ${ }^{24}$ The remaining high-quality sequences were clustered into operational taxonomic units (OTUs) with 97\% similarity by using UPARSE (version 7.0 ${ }^{25}$ and the chimeric sequences were removed by using UCHIME. ${ }^{26}$ The taxonomic assignments of the OTUs were conducted with the RDP classifier ${ }^{27}$ against the SILVA 16S rRNA gene database (Release 128$)^{28}$ with a confidence threshold of 0.70 . The alpha diversity was evaluated by calculating the Shannon diversity index and number of OTUs per sample with the MOTHUR program (version 1.30.1). ${ }^{29}$ Bar plots and heat maps were obtained by using the "vegan" package in $R$ (version 3.3.1). For the beta diversity analysis, a principal coordinates analysis (PCoA) was performed based on the unweighted UniFrac distances by using QIIME (version 1.9). ANOSIM (1000 Monte Carlo permutations) based on the unweighted UniFrac distances was used to compare the similarity of the microbial community between the groups by using the "vegan" package of $R$ (version 3.3.1).

\section{Determination of short-chain fatty acids (SCFAs)}

SCFAs such as acetates, propionates, and butyrates were quantified with an ion chromatograph, as described earlier. ${ }^{30}$ In brief, $0.5 \mathrm{~g}$ of feces samples from the sows and chyme samples from the piglets were weighed, dissolved with $8 \mathrm{~mL}$ ultrapure water, homogenized, and then centrifuged at $5000 \times \mathrm{g}$ for $10 \mathrm{~min}$. The supernatant was diluted $(1: 50)$, filtered through a $0.22 \mu \mathrm{m}$ membrane, and then subjected to SCFA analysis by using an ion chromatography system (Thermo Fisher Scientific, United States).

\section{Statistical analysis}

Data were presented as means \pm SEM. Statistical significance was assessed by means of the independent sample Student's $t$ test using the SPSS v. 20.0 software package (IBM SPSS, USA). Correlations between the differential bacterial genera and physiological parameters were assessed by Spearman correlation analyses. Data were considered to be statistically significant for $P<0.05$ and had a tendency when $0.05<P<0.1$.

\section{Results}

\section{Effects of MFGM supplementation during late gestation on the litter performance of sows}

First, we investigated the effects of MFGM supplementation during late gestation on the litter performance. There was no significant difference in the number of total piglets, live piglets, healthy piglets, weak piglets, stillborn fetuses, total litter weight, average birth weight, and variation in birth weight between the CON and MFGM groups. However, the MFGM group showed a tendency of reduced number of stillborn fetuses as compared to the CON group (Table 1).

\section{Effects of MFGM supplementation during late gestation on plasma parameters}

Then, the effects of MFGM supplementation on plasma parameters were determined to define the metabolic status of the host, as well as the nutrients transfer from the sows to the piglets through umbilical cord blood.

Sow. As shown in Table 2, MFGM supplementation had no effects on the concentrations of TP, GLB, and ALB/GLB ratio $(P>$ $0.05)$, but the concentrations of ALB and CK $(P<0.05)$ increased. In terms of cholesterol and lipoprotein profiles, the concentrations of TC and LDL-C increased in the MFGM group $(P<$ 0.05), but no significant differences in TG, HDL-C, and VLDL-C between the two groups were found. There was no significant difference in GLU and TBA. In addition, the concentration of NEFA in the MFGM group was higher than that in the CON group $(P<0.05)$. 
Table 1 Effects of MFGM supplementation during late gestation on the litter performance of sows

\begin{tabular}{|c|c|c|c|}
\hline Items & CON & MFGM & $P$ value \\
\hline Total piglets $(n)$ & $13.00 \pm 0.63$ & $13.00 \pm 0.65$ & 1.000 \\
\hline Live piglets $(n)$ & $12.38 \pm 0.63$ & $12.86 \pm 0.67$ & 0.608 \\
\hline Weak piglets (birth weight $<1.0 \mathrm{~kg})(n)$ & $0.88 \pm 0.40$ & $0.57 \pm 0.30$ & 0.561 \\
\hline Stillborn fetuses $(n)$ & $0.63 \pm 0.18$ & $0.14 \pm 0.14$ & 0.063 \\
\hline Total litter weight (kg) & $17.72 \pm 0.75$ & $17.74 \pm 0.96$ & 0.989 \\
\hline
\end{tabular}

${ }^{a}$ Coefficient of variation, the ratio of standard deviation of body weight to average body weight of neonatal piglets at birth. MFGM, milk fat globule membrane; CON, CON group fed with the basal diet $(n=11)$; MFGM, MFGM group fed with the basal diet plus MFGM $(n=11)$.

Table 2 Effects of MFGM supplementation during late gestation on the plasma parameters of sows on d 114 of gestation ${ }^{a}$

\begin{tabular}{lcrl}
\hline Items & \multicolumn{1}{c}{ CON } & \multicolumn{1}{l}{ MFGM } & $P$ value \\
\hline TP $\left(\mathrm{g} \mathrm{L}^{-1}\right)$ & $68.91 \pm 0.92$ & $70.40 \pm 0.91$ & 0.266 \\
ALB $\left(\mathrm{g} \mathrm{L}^{-1}\right)$ & $31.48 \pm 0.57$ & $32.91 \pm 0.31$ & 0.042 \\
$\mathrm{GLB}\left(\mathrm{g} \mathrm{L}^{-1}\right)$ & $37.43 \pm 0.91$ & $37.50 \pm 0.92$ & 0.957 \\
ALB $/ \mathrm{GLB}$ & $0.84 \pm 0.03$ & $0.88 \pm 0.03$ & 0.383 \\
$\mathrm{CK}\left(\mathrm{U} \mathrm{L}^{-1}\right)$ & $165.50 \pm 10.20$ & $209.49 \pm 9.85$ & 0.006 \\
TC $\left(\mathrm{mmol} \mathrm{L}^{-1}\right)$ & $1.58 \pm 0.07$ & $1.85 \pm 0.10$ & 0.042 \\
TG $\left(\mathrm{mmol} \mathrm{L}^{-1}\right)$ & $0.50 \pm 0.06$ & $0.59 \pm 0.05$ & 0.248 \\
HDL-C $\left(\mathrm{mmol} \mathrm{L}^{-1}\right)$ & $0.72 \pm 0.03$ & $0.77 \pm 0.03$ & 0.319 \\
LDL-C $\left(\mathrm{mmol} \mathrm{L}^{-1}\right)$ & $0.71 \pm 0.03$ & $0.90 \pm 0.06$ & 0.011 \\
VLDL-C $\left(\mathrm{mmol} \mathrm{L}^{-1}\right)$ & $0.13 \pm 0.02$ & $0.18 \pm 0.03$ & 0.246 \\
GLU $\left(\mathrm{mmol} \mathrm{L}^{-1}\right)$ & $4.97 \pm 0.21$ & $4.72 \pm 0.18$ & 0.389 \\
TBA $\left(\mu \mathrm{mol} \mathrm{L}^{-1}\right)$ & $36.95 \pm 1.98$ & $37.32 \pm 1.24$ & 0.877 \\
NEFA $\left(\mathrm{mmol} \mathrm{L}^{-1}\right)$ & $0.26 \pm 0.01$ & $0.38 \pm 0.02$ & $<0.001$
\end{tabular}

${ }^{a}$ TP, total protein; ALB, albumin; GLB, globulin; CK, creatine kinase; TC, total cholesterol; TG, total triglyceride; HDL-C, high-density lipoprotein cholesterol; LDL-C, low-density lipoprotein cholesterol; VLDL-C, very-low-density lipoprotein cholesterol; GLU, blood glucose; TBA, total bile acid; NEFA, non-esterified fatty acid. MFGM, milk fat globule membrane; CON, CON group fed with the basal diet $(n=11)$; MFGM, MFGM group fed with the basal diet plus MFGM $(n=11)$.

Piglet. Umbilical cord blood is a part of fetal blood circulation. Therefore, we used the umbilical cord blood indexes as plasma parameters of piglets on $\mathrm{d}$ 0. The concentrations of GLU and NEFA in the MFGM group were higher than those in the CON group $(P<0.05)$. In terms of immunoglobulin, the concentration of IgA in the MFGM group was higher than that in the CON group $(P<0.05)$. The concentration of GH in the MFGM group was higher than that in the CON group $(P<0.05)$. In addition, the concentration of IGF-1 did not show a significant difference between the two groups (Table 3). We found no significant difference in the different plasma parameters in piglets on d 21 .

\section{Effects of MFGM supplementation during late gestation on the growth performance of piglets during lactation}

To investigate the transgenerational effects of MFGM supplementation only during late gestation on the growth performance of the offspring, we monitored the dynamic growth of neonatal piglets during lactation. As shown in Table 4, there was no significant difference in the average body weight of the piglets on $\mathrm{d} 7$ after birth between the two groups. However, on d 14 and d 21, the average body weights of the piglets in the

Table 3 Effects of MFGM supplementation during late gestation on the plasma parameters of piglets on $\mathrm{d} 0$ and $\mathrm{d} 21^{a}$

\begin{tabular}{|c|c|c|c|}
\hline Items & $\mathrm{CON}$ & MFGM & $P$ value \\
\hline \multicolumn{4}{|c|}{ Piglets (day 0) $(n=11)$} \\
\hline GLU $\left(\mathrm{mmol} \mathrm{L}^{-1}\right)$ & $0.15 \pm 0.05$ & $0.39 \pm 0.09$ & 0.020 \\
\hline $\operatorname{IgA}\left(\mathrm{g} \mathrm{L}^{-1}\right)$ & $0.96 \pm 0.05$ & $1.10 \pm 0.05$ & 0.020 \\
\hline $\operatorname{IgG}\left(\mathrm{g} \mathrm{L}^{-1}\right)$ & $5.76 \pm 0.12$ & $5.74 \pm 0.28$ & 0.968 \\
\hline $\operatorname{IgM}\left(\mathrm{g} \mathrm{L} \mathrm{L}^{-1}\right)$ & $0.91 \pm 0.08$ & $0.84 \pm 0.07$ & 0.535 \\
\hline $\mathrm{GH}\left(\mathrm{ng} \mathrm{mL}^{-1}\right)$ & $5.34 \pm 0.16$ & $5.92 \pm 0.13$ & 0.011 \\
\hline NEFA $\left(\mathrm{mmol} \mathrm{L}^{-1}\right)$ & $0.25 \pm 0.01$ & $0.21 \pm 0.01$ & 0.005 \\
\hline IGF-1 (ng mL $\left.{ }^{-1}\right)$ & $53.45 \pm 0.42$ & $54.92 \pm 3.01$ & 0.634 \\
\hline \multicolumn{4}{|c|}{ Piglets (day 21) $(n=5)$} \\
\hline GLU $\left(\mathrm{mmol} \mathrm{L}^{-1}\right)$ & $7.33 \pm 0.46$ & $6.38 \pm 0.16$ & 0.080 \\
\hline $\operatorname{IgA}\left(\mathrm{g} \mathrm{L}^{-1}\right)$ & $1.00 \pm 0.09$ & $0.96 \pm 0.05$ & 0.640 \\
\hline $\operatorname{IgG}\left(\mathrm{g} \mathrm{L}^{-1}\right)$ & $7.08 \pm 0.42$ & $7.08 \pm 0.34$ & 0.999 \\
\hline $\operatorname{IgM}\left(\mathrm{g} \mathrm{L} \mathrm{L}^{-1}\right)$ & $1.02 \pm 0.06$ & $0.85 \pm 0.07$ & 0.085 \\
\hline
\end{tabular}

${ }^{a}$ GLU, blood glucose; IgA, immunoglobulin A; IgG, immunoglobulin G; IgM, immunoglobulin M; GH, growth hormone; NEFA, non-esterified fatty acid; IGF-1, insulin-like growth factor-1. MFGM, milk fat globule membrane; CON, CON group fed with the basal diet $(n=11)$; MFGM, MFGM group fed with the basal diet plus $\operatorname{MFGM}(n=11)$.

Table 4 Effects of MFGM supplementation during late gestation on the growth performance of piglets during the neonatal stage ${ }^{a}$

\begin{tabular}{|c|c|c|c|}
\hline Items & $\mathrm{CON}$ & MFGM & $P$ valu \\
\hline \multicolumn{4}{|c|}{ Average body weight (kg) } \\
\hline d 7 & $2.31 \pm 0.11$ & $2.64 \pm 0.13$ & 0.081 \\
\hline d 14 & $3.63 \pm 0.16$ & $4.23 \pm 0.12$ & 0.011 \\
\hline d 21 & $5.49 \pm 0.27$ & $6.23 \pm 0.13$ & 0.033 \\
\hline
\end{tabular}

Average daily weight gain $(\mathrm{g})$

\begin{tabular}{|c|c|c|c|}
\hline $0-7$ & $125.68 \pm 12.53$ & $168.85 \pm 16.07$ & 0.049 \\
\hline d $7-14$ & $199.70 \pm 12.64$ & $241.63 \pm 7.73$ & 0.017 \\
\hline d $14-21$ & $260.00 \pm 10.03$ & $284.33 \pm 13.35$ & 0.176 \\
\hline d $0-21$ & $202.64 \pm 14.66$ & $226.79 \pm 4.26$ & 0.214 \\
\hline
\end{tabular}

${ }^{a}$ MFGM, milk fat globule membrane; CON, CON group fed with the basal diet $(n=11)$; MFGM, MFGM group fed with the basal diet plus $\operatorname{MFGM}(n=11)$. 
Table 5 Effects of MFGM supplementation during late gestation on the intestinal villus morphology of 21 day-old piglets ${ }^{a}$

\begin{tabular}{lll}
\hline Items & CON & MFGM
\end{tabular}

Villus height $(\mu \mathrm{m})$

$\begin{array}{ll}\text { Duodenum } & 416.07 \pm 18.09 \\ \text { Jejunum } & 423.50 \pm 22.58\end{array}$

$473.22 \pm 21.33$

$542.72 \pm 40.14$

$401.05 \pm 34.59$

0.078

0.032

0.263

Crypt depth $(\mu \mathrm{m})$
Duodenum

Jejunum

$184.92 \pm 13.69$

Ileum

$135.43 \pm 10.27$

$102.95 \pm 5.78$

0.003

0.011

0.067

Villus height/crypt depth

$\begin{array}{ll}\text { Duodenum } & 2.32 \pm 0.24 \\ \text { Jejunum } & 3.21 \pm 0.31 \\ \text { Ileum } & 3.71 \pm 0.52\end{array}$

$4.83 \pm 0.42$

$5.48 \pm 0.21$

$5.19 \pm 0.68$ investigated. On d 21 after birth, the villus height of the jejunum in MFGM piglets was higher than that in the CON piglets $(P<0.05)$, and the villus heights of the duodenum and ileum exhibited no significant differences between the two groups. The crypt depths of the duodenum and jejunum were lower in MFGM piglets than those in the CON group $(P<0.05)$, but had no significant difference in the ileum. Villus height/ crypt depth ratios were higher in the MFGM group than those in the CON group $(P<0.05)$, but no significant difference was found in the ileum (Table 5).

Effects of MFGM supplementation during late gestation on tight junctions, mucins, and immune-related genes in the ileal and colonic mucosa of piglets

Subsequently, we investigated the differences in the intestinal barrier functions by analyzing the DAO level and related gene expressions between the CON and MFGM groups. On d 21 after birth, the level of DAO was significantly lower in the piglets of the MFGM group (Fig. 1A), while the relative mRNA expressions of ZO-1, Mucin 20, and TLR2 in the MFGM group were significantly higher in the ileal mucosa (Fig. 1B-D).

In the colonic mucosa of the 21 day-old piglets, maternal MFGM supplementation significantly upregulated the relative mRNA expressions of Occludin, Claudin-1, Claudin-2, Claudin4, and ZO-1 (Fig. 1E). With regard to mucins, the relative mRNA expressions of Mucin 2, Mucin 4, and Mucin 13 in the MFGM group were higher than those of the CON group $(P<0.05)$. The relative mRNA expression of Mucin 1 showed an increasing tendency, while the expression of Mucin 20 showed no significant difference (Fig. 1F). In terms of immune-related genes, the relative mRNA expressions of TNF- $\alpha$, INF- $\gamma$, IL-22, TLR2, and

Furthermore, the small intestinal morphological structures of 21 day-old piglets in the MFGM and CON groups were

Effects of MFGM supplementation during late gestation on the morphological structure of small intestinal epithelium in piglets

(1)

A

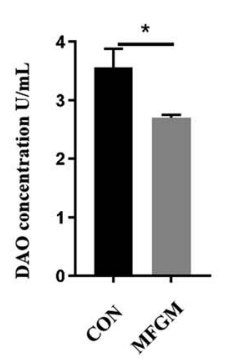

B
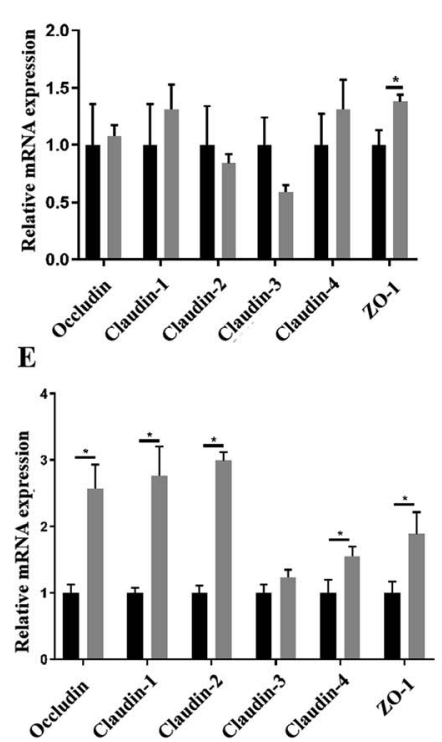

C
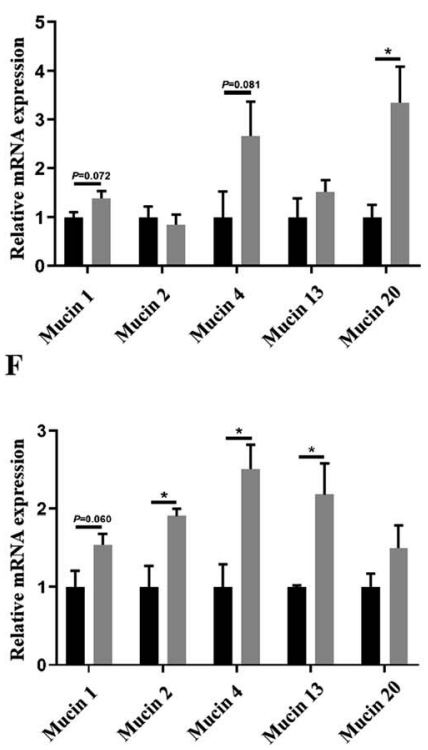

D
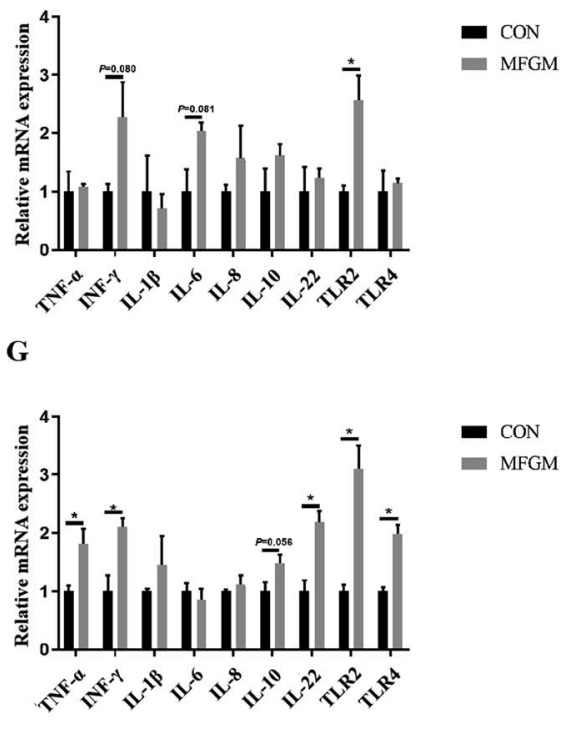

Fig. 1 DAO level in plasma (A), relative mRNA expressions of tight junctions, mucins, and immune-related genes in the ileal mucosa (B-D) and colonic mucosa (E-G) of 21 day-old piglets ( $n=5$ /group). ZO- 1 , zonulin-1; TNF- $\alpha$, tumor necrosis factor- $\alpha$; INF- $\gamma$, interferon- $\gamma$; IL-1 $\beta$, interleukin-13; IL-6, interleukin-6; IL-8, interleukin-8; IL-10, interleukin-10; IL-22, interleukin-22, TLR2, toll-like receptor 2; TLR4, toll-like receptor 4. CON, CON group fed with the basal diet; MFGM, MFGM group fed with the basal diet plus MFGM. Data are represented as means \pm SEM. *, $P<$ 0.05 . 
A

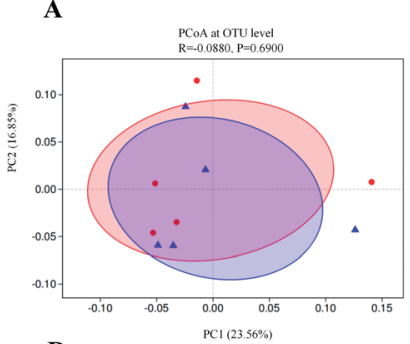

D

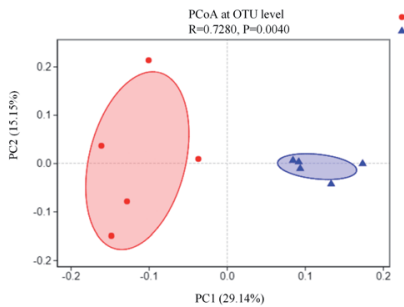

B

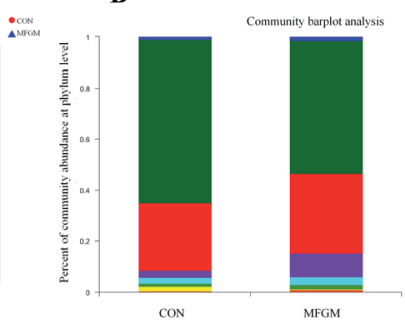

E

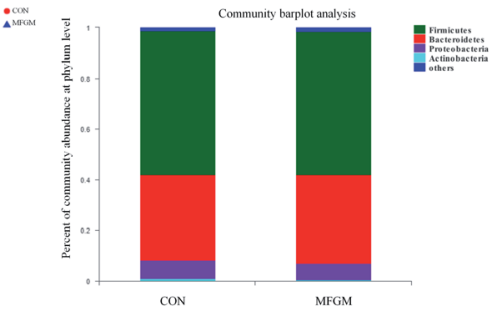

C

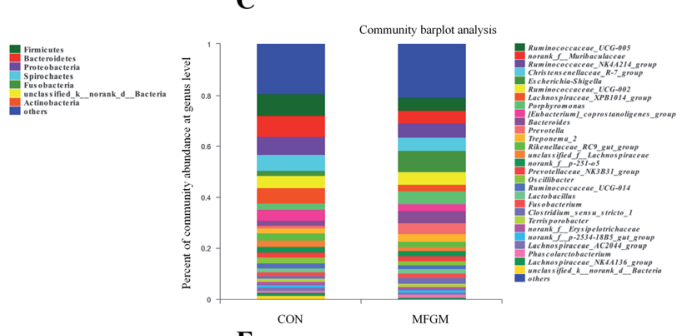

$\mathbf{F}$

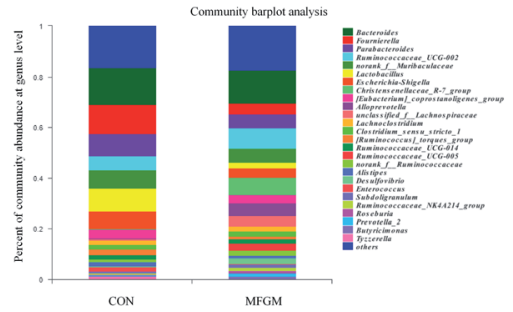

Fig. 2 PCoA: composition of the fecal microbiota at the phylum and genus levels of sows ( $n=5 /$ group) on d 114 of gestation (A-C) and 21 dayold piglets ( $n=5 /$ group) (D-F). CON, CON group fed with the basal diet; MFGM, MFGM group fed with the basal diet plus MFGM.

TLR4 in the MFGM group were higher than those in the CON group $(P<0.05)$. The relative mRNA expression of IL-10 tended to increase in MFGM piglets, but no significant difference was observed in the IL-1 $\beta$, IL-6, and IL-8 levels (Fig. 1G).

\section{Effects of MFGM supplementation during late gestation on the fecal microbiota of sows and piglets}

To investigate the effects of MFGM supplementation during late gestation on the fecal microbial community of sows and neonatal piglets, the microbiota composition and diversity were assessed by the deep sequencing of the $\mathrm{V} 3-\mathrm{V} 4$ region of the $16 \mathrm{~S}$ rRNA genes.

Sow. A total of 1026190 high-quality 16S rRNA gene sequences were generated from 10 feces samples. Based on $97 \%$ sequence similarity, 1537 OTUs were identified and then assigned to 18 phyla, 26 classes, 50 orders, 96 families, 299 genera, and 605 species. The microbial alpha diversity in the feces of both the groups is shown in ESI Table S5. $\uparrow$ The Sobs, Ace, Shannon, Simpson, and Chao indexes in the fecal microbiota of both the groups exhibited no significant differences $(P>$ 0.05). In addition, PCoA based on unweighted UniFrac distances revealed that the fecal microbial community structure was different between the two groups (Fig. 2A). The presence of phyla and genera in relative abundance in the fecal microbiota is evident (Fig. 2B and C). Firmicutes and Bacteroidetes were the most dominant phyla in the fecal microbiota of the MFGM and CON groups, followed by Proteobacteria and Spirochaetes, while other phyla were present at very low relative abundances. At the genus level, Ruminococcaceae_UCG-005, norank $f$ _Muribaculaceae, and Ruminococcaceae_NK4A214_group were the major genera. Further, we found that the abundance of Prevotella in the sow feces in the MFGM group was higher than that in the CON group via a significance test of the intergroup differences $(P<0.05)$, while the abundance of norank $f \_$Muribaculaceae and Lachnospiraceae_XPB1014_group was lower than those in the CON group $(P<0.05)$ (Fig. 3A).
Piglet. From 21 day-old piglet feces samples, 390930 highquality 16S rRNA gene sequences were generated. Based on 97\% sequence similarity, 911 OTUs were identified and then assigned to 19 phyla, 25 classes, 50 orders, 100 families, 296 genera, and 532 species. The alpha diversity values showed that the Sobs, Shannon, Simpson, Ace, and Chao indexes in the MFGM piglets' feces were higher than those in the CON group $(P$

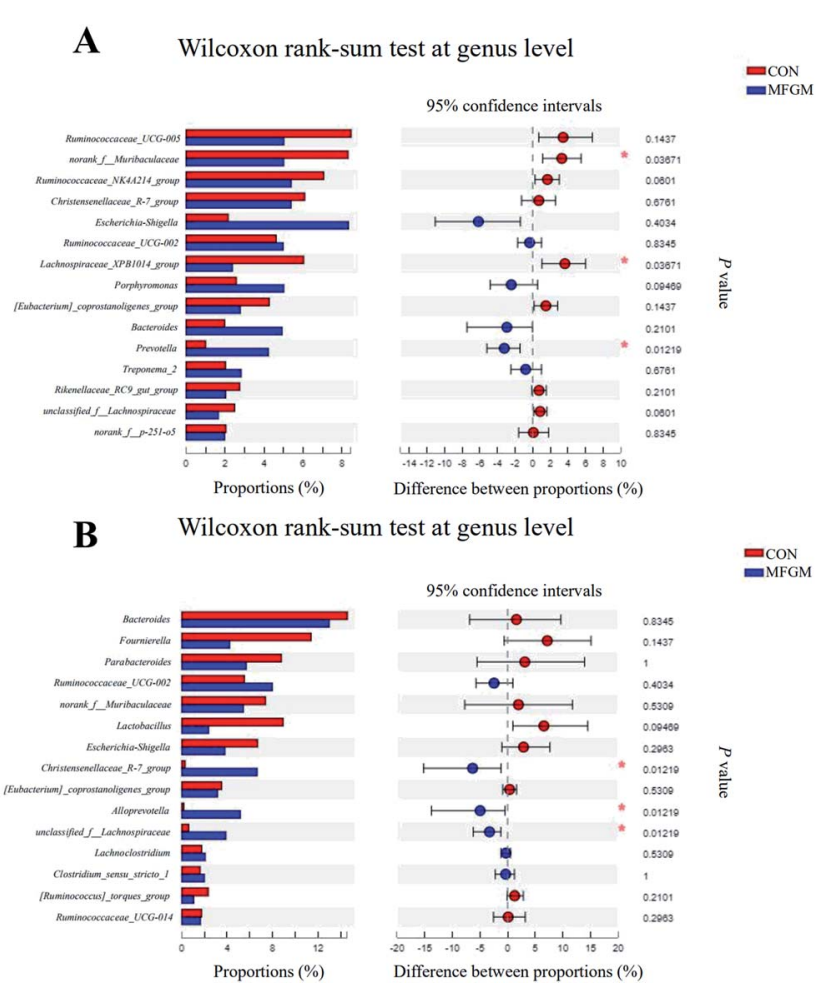

Fig. 3 Microbial comparisons at the genus level of the sows $(n=5$ / group) on d 114 of gestation (A) and 21 day-old piglets ( $n=5 /$ group) (B). CON, CON group fed with the basal diet; MFGM, MFGM group fed with the basal diet plus MFGM. *, $P<0.05$. 
Table 6 Effects of MFGM supplementation during late gestation on the SCFAs of sows on d 114 of gestation and 21 day-old piglets ${ }^{a}$

\begin{tabular}{llll}
\hline Items & CON & MFGM & $P$ value \\
\hline
\end{tabular}

\section{Sows' feces $\left(\mathrm{mg} \mathrm{kg}^{-1}\right)$}

Acetate $\quad 2332.16 \pm 247.67$

Propionate $\quad 791.45 \pm 22.58$

Butyrate $\quad 285.65 \pm 60.04$

\section{$3790.45 \pm 393.28$ \\ $1372.13 \pm 212.48$ \\ $385.99 \pm 100.01$}

0.037

0.006

0.038

Piglets' ileum ( $\left.\mathrm{mg} \mathrm{kg}^{-1}\right)$

$\begin{array}{lrl}\text { Acetate } & 479.05 & \pm 83.62 \\ \text { Propionate } & 127.23 & \pm 50.12 \\ \text { Butyrate } & 8.66 & \pm 1.10\end{array}$

$507.43 \pm 42.39$

$271.92 \pm 35.93$

$8.46 \pm 4.89$

0.789

0.047

0.967

Piglets' colon ( $\left.\mathrm{mg} \mathrm{kg}^{-\mathbf{1}}\right)$

\begin{tabular}{|c|c|c|c|}
\hline Ac & $1090.18 \pm 155.85$ & $1020.60 \pm 326.03$ & 0.854 \\
\hline Propionate & $359.15 \pm 22.61$ & $358.30 \pm 101.01$ & 0.994 \\
\hline Butyrate & $69.09 \pm 16.23$ & $71.74 \pm 55.64$ & .96 \\
\hline
\end{tabular}

${ }^{a}$ MFGM, milk fat globule membrane; CON, CON group fed with the basal diet $(n=5)$; MFGM, MFGM group fed with the basal diet plus $\operatorname{MFGM}(n=5)$.

$<0.05$ ), indicating that the microbial diversity and richness in piglets' feces were significantly elevated by the MFGM treatment. PCoA based on unweighted UniFrac distances showed that the fecal microbial community structure was different between the two groups $(R=0.728, P<0.05)$ (Fig. 2D). Firmicutes, Bacteroidetes, and Fusobacteria were the main phyla in both the groups (Fig. 2E). Bacteroides, Lachnoclostridium, and Ruminococcaceae_UCG-002 were the main genera in both the groups (Fig. 2F). Differential analysis of the bacteria between the two groups at the genus level revealed that the abundance of Christensenellaceae_R-7_group, Alloprevotella, and unclassied_$f$ _Lachnospiraceae in the feces of MFGM piglets was higher than those in the CON piglets $(P<0.05)$ (Fig. 3B).

Effects of MFGM supplementation during late gestation on the fecal SCFAs levels and mRNA expressions of intestinal G protein-coupled receptors (GPRs)

To investigate the metabolic status of the microbiota, we analyzed the fecal concentrations of SCFAs in the sows, as well as their concentrations in the ileum and colonic chyme of piglets and relative mRNA expressions of GPRs.

Sow. The results of the fecal SCFAs are listed in Table 6 . When compared with the CON group, the concentrations of acetate and propionate in the MFGM group were higher $(P<$ $0.05)$. The concentration of butyrate did not differ significantly between the two groups.

Piglet. In the ileum, the concentration of propionate in the MFGM group was higher than that in the CON group $(P<0.05)$. In the colon, the concentrations of acetate, propionate, and butyrate were not significantly different between the two groups (Table 6). In terms of GPRs, the relative mRNA expression of GPR41 in the ileal mucosa of piglets in the MFGM group was higher than that in the CON group (Fig. 4A). Relative mRNA expressions of GPR41, GPR43, and GPR120 in the colonic mucosa in the MFGM group were higher than those in the CON group, with no difference in GPR119 (Fig. 4B).

\section{Correlation analysis between differential bacterial genera and physiological parameters}

Eventually, the correlations between the critical differential bacterial genera and differential parameters were analyzed, as shown in Fig. 5.

Sow. Prevotella was positively correlated with the concentrations of GH $(R=0.693, P<0.05)$, NEFA $(R=0.758, P<0.05)$, TC $(R=0.673, P<0.05)$, and LDL-C $(R=0.783, P<0.05)$. Norank $f$ _Muribaculaceae was negatively correlated with the concentrations of TC $(R=-0.648, P<0.05)$, LDL-C $(R=-0.640$, $P<0.05)$, and IgA $(R=-0.717, P<0.05)$. Lachnospiraceae_XPB1014_group was negatively correlated with the concentrations of GH $(R=-0.827, P<0.05)$, NEFA $(R=-0.648$, $P<0.05)$, TC $(R=-0.685, P<0.05)$, LDL $(R=-0.762, P<0.05)$, and CK $(R=-0.479, P<0.05)$ (Fig. 5A).

Piglet. We analyzed the correlation between the critical differential bacteria genera in piglet feces and differentially expressed the indexes in the colonic mucosa. Christensenellaceae_R-7_group was positively correlated with the relative mRNA expressions of Occludin $(R=0.855, P<0.05)$, Mucin $13(R=$ $0.818, P<0.05)$, TLR4 $(R=0.927, P<0.05)$, Claudin-2 $(R=0.766$,
$\mathbf{A}$

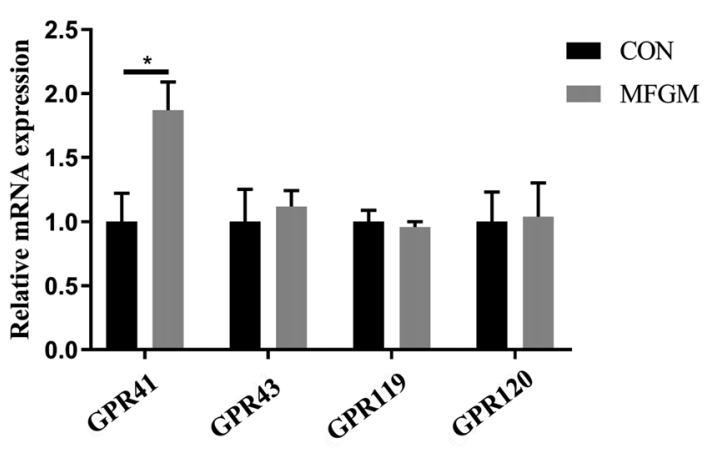

B

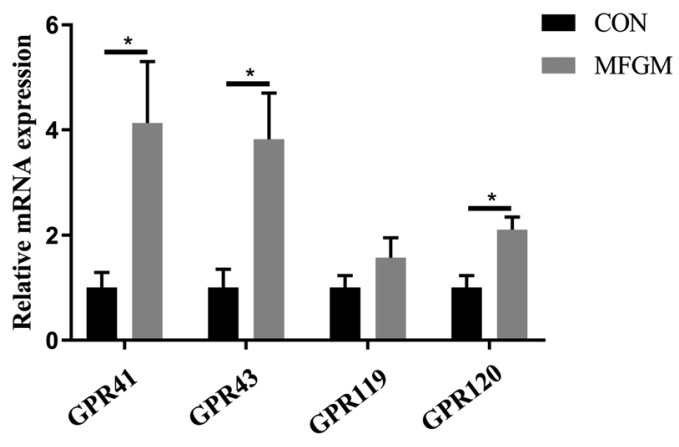

Fig. 4 Relative mRNA expressions of GPRs in the ileal mucosa (A) and colonic mucosa (B) of 21 day-old piglets ( $n=5 /$ group). GPR, G proteincoupled receptor. CON, CON group fed with the basal diet; MFGM, MFGM group fed with the basal diet plus MFGM. Data are represented as means \pm SEM. * $P<0.05$. 
A

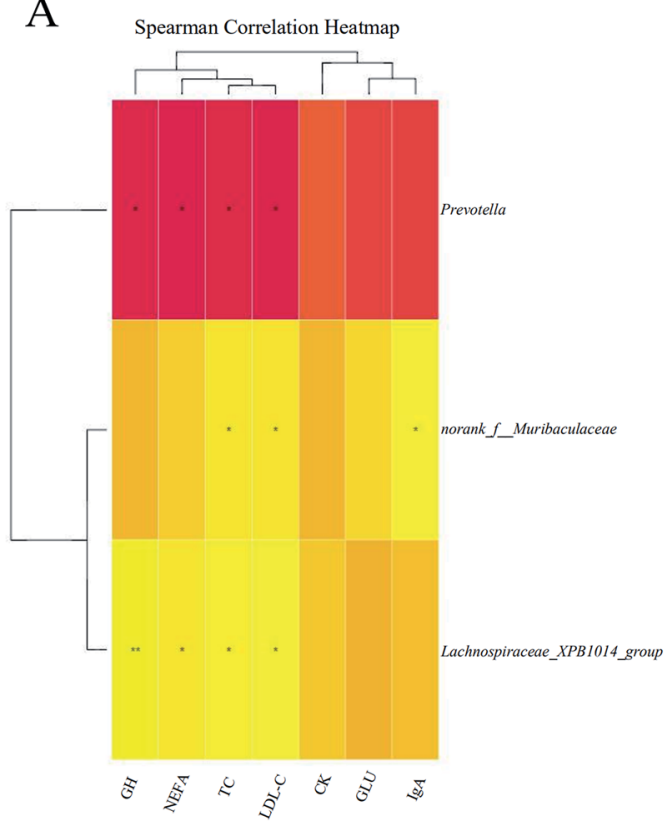

B

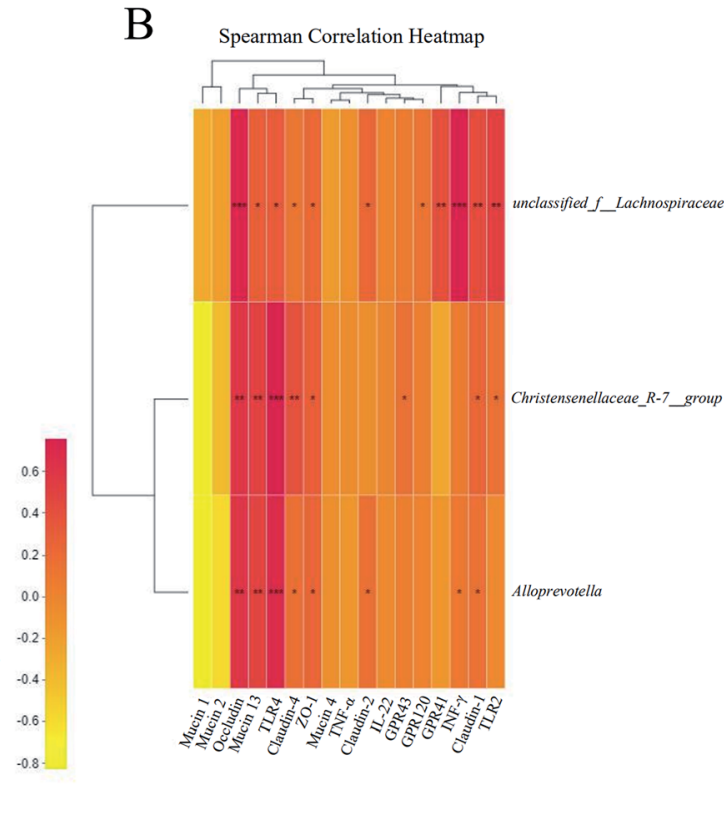

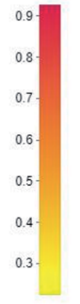

Fig. 5 Spearman correlation analysis between differential bacterial genera and differential parameters of the sows ( $n=5 / \mathrm{group}$ ) on d 114 of gestation (A) and 21 day-old piglets ( $n=5 /$ group) (B). ZO-1: zonulin- 1 ; TNF- $\alpha$ : tumor necrosis factor- $\alpha$; INF- $\gamma$ : interferon- $\gamma$; IL-1 $\beta$ : interleukin-1 $\beta$; IL-6: interleukin-6; IL-8: interleukin-8; IL-10: interleukin-10; IL-22: interleukin-22, TLR2: toll-like receptor 2; TLR4: toll-like receptor 4. CON, CON group fed with the basal diet; MFGM, MFGM group fed with the basal diet plus MFGM. $-0.8-0.6,0.3$, and -0.9 , correlation coefficients. *, $P$ $<0.05$.

$P<0.05)$, IL-22 $(R=0.721, P<0.05)$, GPR43 $(R=0.661, P<0.05)$, Claudin-1 $(R=0.700, P<0.05)$, and TLR2 $(R=0.661, P<0.05)$. Alloprevotella was positively correlated with the relative mRNA expressions of Occludin $(R=0.855, P<0.05)$, Mucin $13(R=$ $0.806, P<0.05)$, TLR4 $(R=0.891, P<0.05)$, Claudin-2 $(R=0.669$, $P<0.05)$, IL-22 $(R=0.697, P<0.05)$, Claudin-4 $(R=0.663, P<$ $0.05)$, INF- $\gamma(R=0.636, P<0.05)$, and TLR $2(R=0.576, P<0.05)$. Unclassied_f_Lachnospiraceae was found to positively correlate with the relative mRNA expressions of Occludin $(R=0.915, P<$ $0.001)$, Mucin $13(R=0.721, P<0.05)$, TLR4 $(R=0.733, P<0.05)$, Claudin-2 $(R=0.644, P<0.05)$, IL-22 $(R=0.685, P<0.05)$, Claudin-4 $(R=0.693, P<0.05)$, GPR120 $(R=0.648, P<0.05)$, GPR41 $(R=0.770, P<0.05)$, INF- $\gamma(R=0.927, P<0.05)$, Claudin$1(R=0.782, P<0.05)$, and TLR2 $(R=0.818, P<0.05)$.

\section{Discussion}

Nutritional strategies of the sows during late gestation deserve attention due to their extensive metabolic changes and rapid growth in their fetuses. In the present study, we investigated the effects of MFGM supplementation during late gestation on sows and their offspring. Our results revealed that dietary MFGM supplementation during gestation had a positive impact on the growth and development of their piglets during the neonatal stage by improving the plasma parameters, intestinal barrier, and fecal microbiota.

In our study, there was no significant difference in the litter performance between the CON and MFGM groups. However, the MFGM group had a tendency to reduce the number of stillborn fetuses. This may be related to the improvement in the plasma parameters in the MFGM sows, particularly the increases in ALB, LDL-C, and NEFA concentrations. ALB plays an important role in the maintenance of plasma pressure and nutrient balance. ${ }^{31}$ The increased ALB concentration in the MFGM group could be beneficial toward the health of sows. The increased LDL-C and NEFA concentrations revealed that accelerated fatty acid synthesis can provide more dominant fatty acids for homeostatic metabolism. ${ }^{32-34}$ At the same time, MFGM supplementation increased the abundance of Prevotella, which has been shown to be associated with improved reproductive performance and fatty acid synthesis. ${ }^{35}$ Further, a correlation analysis showed that Prevotella was positively correlated with LDL-C and NEFA concentrations in sows' plasma and GH concentration in umbilical cord blood. An earlier study showed that MFGM supplementation can have a positive impact on the host by regulating its gut microbiota. ${ }^{16}$ Subsequently, we found that the concentrations of acetate, propionate, and butyrate in the feces of MFGM sows were significantly higher. An earlier study showed that the SCFAs produced by the maternal intestinal flora can enter the embryo via the blood flow and exert on the GPRs, shaping the development of the metabolic system of the embryo and protecting the metabolic health of the offspring. ${ }^{36}$ Umbilical cord blood-the main pathway for the fetus to absorb nutrients from the mother-is crucial for the growth and development of the fetus. We found that the concentrations of GH, IgA, GLU, and NEFA in the umbilical cord blood in the MFGM group were significantly higher than those in the CON group. Evidently, GH is critical for the growth and development of fetuses, and IgA in the umbilical cord blood is crucial for the intestinal development and immune maturation 
of the fetus after birth. ${ }^{37}$ In addition, increased concentrations of GLU and NEFA in the umbilical cord blood of the MFGM group could also provide more nutrition for the fetuses.

Next, we investigated the effects of MFGM supplementation into sows' diet during late gestation only on the growth performance and intestinal microecology of their neonatal piglets. The average body weight of 21 day-old piglets in the MFGM group was significantly higher than that in the CON group, indicating that MFGM supplementation during late gestation could improve the growth performance of the offspring in the neonatal stage. Early microbial colonization plays an important role in the maintenance of metabolic homeostasis and development of the immune system in the offspring, ${ }^{38}$ while maternal intestinal strains function as the primary source for the neonatal microbiome. ${ }^{10}$ In the feces of 21 day-old MFGM piglets, we found that the relative abundance of Christensenellaceae_R-7_group was reported to be beneficial to the health ${ }^{39}$ and intestinal functions ${ }^{40}$ and is highly heritable; ${ }^{41}$ further, it was significantly higher than that in CON piglets. Therefore, the higher abundance of Christensenellaceae_R7 group in MFGM piglets contributed toward the enhanced growth performance.

Our further analysis revealed that MFGM supplementation into sows' diet only during late gestation could also improve the morphological structure and barrier functions of the intestines in 21 day-old MFGM piglets. In particular, the villus height in the jejunum increased significantly, while the crypt depth in the duodenum and jejunum decreased significantly; further, the relative mRNA expressions of the tight junctions (Occludin, Claudin-1, Claudin-2, Claudin-4, and ZO-1) and mucins (Mucin 2, Mucin 4, Mucin 13, and Mucin 20) increased significantly. Moreover, the increased TNF- $\alpha$ and INF- $\gamma$ expressions indicated an improvement in the immunomodulatory activity. ${ }^{42}$ IL-22 plays an important role in mediating colon health. ${ }^{43}$ TLR2 and TLR4, one of the earliest determinants of immune activation, are involved in immune recognition and signal transduction. ${ }^{\mathbf{4}}$ Increased expressions of these immune-related genes enhanced the intestinal immunological barrier. Early intestinal development in mammals is important for the growth and health of neonates. ${ }^{45,46}$ A modified morphological structure and enhanced barrier functions are considered to be the main aspects of intestinal function improvement. ${ }^{47} \mathrm{GH}$ concentration in umbilical cord blood was significantly higher in the MFGM group than that in the CON group. This might be one of the reasons for the improved intestinal development of neonatal piglets since the nutritional and protective effects of $\mathrm{GH}$ on the small intestine have been proven in earlier studies, ${ }^{48}$ as well as its ability to promote the villi development ${ }^{49}$ and inhibit LPSinduced intestinal barrier destruction. ${ }^{50}$ In addition, many studies have shown a link between the gut microbiota and intestinal barrier. ${ }^{51,52}$ Zhang et al. (2019) revealed a positive correlation between four Lachnospiraceae-related OTUs and Occludin expression. ${ }^{51}$ The positive correlation between unclassified_f_Lachnospiraceae and Occludin was also found in the present study. At the same time, the composition of the gut microbiota is important for the host because it can not only directly affect the host but also interact with the host via its

metabolites (mainly SCFAs), ${ }^{53,54}$ which play important roles in the antioxidant, antiinflammatory, balance of gut microbiota, and intestinal function. ${ }^{55,56}$ Christensenellaceae_R-7_group is considered to be a butyrate producer, playing a key role in the intestinal environment and immunomodulation. ${ }^{40}$ It has been reported that butyrates can induce the relative mRNA expression of Mucin 2 and its secretion in goblet-like cells, ${ }^{57}$ as well as promote the assembly of Occludin through the AMPK pathway. ${ }^{52}$ In addition, the propionate concentration in the ileal chyme; GPR41 expression in the ileal mucosa; and GPR41, GPR43, and GPR120 expressions in the colonic mucosa in MFGM piglets were significantly higher than those in the CON group. Since there was no difference in the concentration of colonic SCFAs, we concluded that unclassified_$f \_$Lachnospiraceae and Christensenellaceae_R-7_group induced an increase in the GPR expressions according to the heat map. Moreover, it was reported that TNF- $\alpha$ could induce the overexpression of GPR120.8 ${ }^{58}$ GP43 was closely regulated with the receptors that are important for innate immunity, such as tolllike receptors (TLR2 and TLR4). ${ }^{59}$ The increased relative mRNA expressions of GPRs in MFGM piglets could subsequently enhance the combining ability of intestinal epithelial cells with SCFAs to further regulate the immunological function and improve the intestinal barrier. GPR43 regulates the proliferation of colonic group 3 innate lymphoid cells and IL-22 production. ${ }^{60}$ GPR120 could mediate the TNF- $\alpha$ expression. ${ }^{61}$ This partly explains why the expressions of immune-related genes were increased in the intestinal barrier of MFGM piglets. Therefore, we concluded that MFGM supplementation during late gestation could improve the growth performance of neonatal piglets partly by modifying the intestinal functions and microecology.

\section{Conclusion}

In conclusion, MFGM supplementation during late gestation improved the plasma parameters and intestinal microbiota of sows. The increased GH, IgA, GLU, and NEFA transfers through the umbilical cord blood and the transgenerational imprinting of microbiota colonization, particularly Christensenellaceae_R7_group in neonatal piglets, promoted their intestinal development and barrier functions, thereby improving their growth performance during lactation. These findings provide a new option for the nutritional intervention of neonates through maternal dietary manipulation.

\section{Abbreviations}

MFGM

LPS

TP

ALB

GLB

CK

TC

TG
Milk fat globule membrane

Lipopolysaccharides

Total protein

Albumin

Globulin

Creatine kinase

Serum total cholesterol

Total triglyceride 


$\begin{array}{ll}\text { HDL-C } & \text { High-density lipoprotein cholesterol } \\ \text { LDL-C } & \text { Low-density lipoprotein cholesterol } \\ \text { VLDL-C } & \text { Very-low-density lipoprotein cholesterol } \\ \text { GLU } & \text { Blood glucose } \\ \text { TBA } & \text { Total bile acid } \\ \text { NEFA } & \text { Non-esterified fatty acid } \\ \text { GH } & \text { Growth hormone } \\ \text { IGF-1 } & \text { Insulin-like growth factors } \\ \text { DAO } & \text { Diamine oxidase } \\ \text { IgA } & \text { Immunoglobulin A } \\ \text { IgG } & \text { Immunoglobulin G } \\ \text { IgM } & \text { Immunoglobulin M } \\ \text { GADPH } & \text { Glyceraldehyde-3-phosphate dehydrogenase } \\ \text { SCFA } & \text { Short-chain fatty acid } \\ \text { GPR } & \text { G protein-coupled receptor } \\ \text { ZO-1 } & \text { Zonulin-1 } \\ \text { TNF- } \alpha & \text { Tumor necrosis factor- } \alpha \\ \text { INF- } \gamma & \text { Interferon- } \gamma \\ \text { IL-1 } \beta & \text { Interleukin-1 } \beta \\ \text { IL-6 } & \text { Interleukin-6 } \\ \text { IL-8 } & \text { Interleukin-8 } \\ \text { IL-10 } & \text { Interleukin-10 } \\ \text { IL-22 } & \text { Interleukin-22 } \\ \text { TLR2 } & \text { Toll-like receptor } 2 \\ \text { TLR4 } & \text { Toll-like receptor } 4 \\ & \end{array}$

\section{Conflicts of interest}

The authors declare no conflicts on financial, personal or professional interests for the manuscript.

\section{Acknowledgements}

The materials of milk fat globule membrane were provided by the Sanyuan Foods Co. Ltd. in Beijing, China. We appreciated the experimental animal platform of China Agricultural University in Hebei, China, for the assistance in this study. This research was financially supported by the Beijing Municipal Natural Science Foundation (S170001), the National Natural Science Foundation of China (31630074 and 31902170), the National Key Research and Development Program of China (2016YFD0500506 and 2018YDF0501002), the China Agriculture Research System (CARS-35), the 111 Project (B16044) and Jinxinnong Animal Science Developmental Foundation.

\section{References}

1 J. Wang, Z. Wu, D. Li, N. Li, S. V. Dindot, M. C. Satterfield, F. W. Bazer and G. Wu, Nutrition, epigenetics, and metabolic syndrome, Antioxid. Redox Sign., 2012, 17, 282301.

2 R. N. Funston, D. M. Larson and K. A. Vonnahme, Effects of maternal nutrition on conceptus growth and offspring performance: implications for beef cattle production, $J$. Anim. Sci., 2010, 88, E205-E215.

3 H. Chen, D. Chen, W. Qin, Y. Liu, L. Che, Z. Huang, Y. Luo, Q. Zhang, D. Lin, Y. Liu, G. Han, S. DeSmet and J. Michiels,
Wheat bran components modulate intestinal bacteria and gene expression of barrier function relevant proteins in a piglet model, Int. J. Food Sci. Nutr., 2017, 68, 65-72.

4 J. B. Ewaschuk, G. K. Murdoch, I. R. Johnson, K. L. Madsen and C. J. Field, Glutamine supplementation improves intestinal barrier function in a weaned piglet model of Escherichia coli infection, Br. J. Nutr., 2011, 106, 870-877.

5 M. Cheng, X. Zhang, J. Zhu, L. Cheng, J. Cao, Z. Wu, P. Weng and X. Zheng, A metagenomics approach to the intestinal microbiome structure and function in high fat dietinduced obesity mice fed with oolong tea polyphenols, Food Funct., 2018, 9, 1079-1087.

6 G. Clarke, R. M. Stilling, P. J. Kennedy, C. Stanton, J. F. Cryan and T. G. Dinan, Minireview: Gut microbiota: the neglected endocrine organ, Mol. Endocrinol., 2014, 28, 1221-1238.

7 J. Wang, H. Tang, C. Zhang, Y. Zhao, M. Derrien, E. Rocher, J. E. van-Hylckama Vlieg, K. Strissel, L. Zhao, M. Obin and J. Shen, Modulation of gut microbiota during probioticmediated attenuation of metabolic syndrome in high fat diet-fed mice, ISME J., 2015, 9, 1-15.

8 R. C. Anderson, A. L. Cookson, W. C. McNabb, Z. Park, M. J. McCann, W. J. Kelly and N. C. Roy, Lactobacillus plantarum MB452 enhances the function of the intestinal barrier by increasing the expression levels of genes involved in tight junction formation, BMC Microbiol., 2010, 10, 316.

9 J. Hu, L. Chen, W. Zheng, M. Shi, L. Liu, C. Xie, X. Wang, Y. Niu, Q. Hou, X. Xu, B. Xu, Y. Tang, S. Zhou, Y. Yan, T. Yang, L. Ma and X. Yan, Lactobacillus frumenti facilitates intestinal epithelial barrier function maintenance in early-weaned piglets, Front. Microbiol., 2018, 9, 897.

10 P. Ferretti, E. Pasolli, A. Tett, F. Asnicar, V. Gorfer, S. Fedi, F. Armanini, D. T. Truong, S. Manara, M. Zolfo, F. Beghini, R. Bertorelli, V. De Sanctis, I. Bariletti, R. Canto, R. Clementi, M. Cologna, T. Crifo, G. Cusumano, S. Gottardi, C. Innamorati, C. Mase, D. Postai, D. Savoi, S. Duranti, G. A. Lugli, L. Mancabelli, F. Turroni, C. Ferrario, C. Milani, M. Mangifesta, R. Anzalone, A. Viappiani, M. Yassour, H. Vlamakis, R. Xavier, C. M. Collado, O. Koren, S. Tateo, M. Soffiati, A. Pedrotti, M. Ventura, C. Huttenhower, P. Bork and N. Segata, Mother-to-infant microbial transmission from different body sites shapes the developing infant gut microbiome, Cell Host Microbe, 2018, 24, 133-145 e135.

11 J. L. McManaman and M. C. Neville, Mammary physiology and milk secretion, Adv. Drug Delivery Rev., 2003, 55, 629641.

12 J. L. McManaman, T. D. Russell, J. Schaack, D. J. Orlicky and H. Robenek, Molecular determinants of milk lipid secretion, J. Mammary Gland Biol. Neoplasia, 2007, 12, 259-268.

13 D. Ramiro-Cortijo, P. Singh, Y. Liu, E. Medina-Morales, W. Yakah, S. D. Freedman and C. R. Martin, Breast milk lipids and fatty acids in regulating neonatal intestinal development and protecting against intestinal injury, Nutrients, 2020, 12, 534. 
14 R. C. Sprong, M. F. Hulstein, T. T. Lambers and R. van der Meer, Sweet buttermilk intake reduces colonisation and translocation of Listeria monocytogenes in rats by inhibiting mucosal pathogen adherence, Br. J. Nutr., 2012, 108, 2026-2033.

15 D. R. Snow, R. E. Ward, A. Olsen, R. Jimenez-Flores and K. J. Hintze, Membrane-rich milk fat diet provides protection against gastrointestinal leakiness in mice treated with lipopolysaccharide, J. Dairy Sci., 2011, 94, 2201-2212.

16 T. Li, J. Gao, M. Du and X. Mao, Milk fat globule membrane supplementation modulates the gut microbiota and attenuates metabolic endotoxemia in high-fat diet-fed mice, J. Funct. Foods, 2018, 47, 56-65.

17 S. Huang, Z. Wu, C. Liu, D. Han, C. Feng, S. Wang and J. Wang, Milk fat globule membrane supplementation promotes neonatal growth and alleviates inflammation in low-birth-weight mice treated with lipopolysaccharide, BioMed Res. Int., 2019, 2019, 4876078.

18 T. Song, J. Peng, J. Ren, H. K. Wei and J. Peng, Cloning and characterization of spliced variants of the porcine $\mathrm{G}$ protein coupled receptor 120, BioMed Res. Int., 2015, 2015, 813816.

19 L. Xia, Y. Yang, J. Wang, Y. Jing and Q. Yang, Impact of TGEV infection on the pig small intestine, Virol. J., 2018, 15, 102.

20 Y. Li, J. Wang, Y. Li, H. Wu, S. Zhao and Q. Yu, Protecting intestinal epithelial cells against deoxynivalenol and E. coli damage by recombinant porcine IL-22, Vet. Microbiol., 2019, 231, 154-159.

21 G. Li, H. Su, Z. Zhou and W. Yao, Identification of the porcine $\mathrm{G}$ protein-coupled receptor 41 and 43 genes and their expression pattern in different tissues and development stages, PLoS One, 2014, 9, e97342.

22 W. Ren, P. Wang, J. Yan, G. Liu, B. Zeng, T. Hussain, C. Peng, J. Yin, T. Li, H. Wei, G. Zhu, R. J. Reiter, B. Tan and Y. Yin, Melatonin alleviates weanling stress in mice: Involvement of intestinal microbiota, J. Pineal Res., 2018, 64, e12448.

23 J. G. Caporaso, C. L. Lauber, W. A. Walters, D. Berg-Lyons, J. Huntley, N. Fierer, S. M. Owens, J. Betley, L. Fraser, M. Bauer, N. Gormley, J. A. Gilbert, G. Smith and R. Knight, Ultra-high-throughput microbial community analysis on the Illumina HiSeq and MiSeq platforms, ISME J., 2012, 6, 1621-1624.

24 A. P. Masella, A. K. Bartram, J. M. Truszkowski, D. G. Brown and J. D. Neufeld, PANDAseq: paired-end assembler for illumina sequences, BMC Bioinf., 2012, 13, 31.

25 R. C. Edgar, UPARSE: highly accurate OTU sequences from microbial amplicon reads, Nat. Methods, 2013, 10, 996-998.

26 R. C. Edgar, B. J. Haas, J. C. Clemente, C. Quince and R. Knight, UCHIME improves sensitivity and speed of chimera detection, Bioinformatics, 2011, 27, 2194-2200.

27 G. Bacci, A. Bani, M. Bazzicalupo, M. T. Ceccherini, M. Galardini, P. Nannipieri, G. Pietramellara and A. Mengoni, Evaluation of the performances of ribosomal database project (RDP) classifier for taxonomic assignment of $16 \mathrm{~S}$ rRNA metabarcoding sequences generated from Illumina-Solexa NGS, Journal of Genomics, 2015, 3, 36-39.
28 E. Pruesse, C. Quast, K. Knittel, B. M. Fuchs, W. Ludwig, J. Peplies and F. O. Glockner, SILVA: a comprehensive online resource for quality checked and aligned ribosomal RNA sequence data compatible with ARB, Nucleic Acids Res., 2007, 35, 7188-7196.

29 P. D. Schloss, S. L. Westcott, T. Ryabin, J. R. Hall, M. Hartmann, E. B. Hollister, R. A. Lesniewski, B. B. Oakley, D. H. Parks, C. J. Robinson, J. W. Sahl, B. Stres, G. G. Thallinger, D. J. Van Horn and C. F. Weber, Introducing mothur: open-source, platform-independent, community-supported software for describing and comparing microbial communities, Appl. Environ. Microbiol., 2009, 75, 7537-7541.

30 B. He, Y. Bai, L. Jiang, W. Wang, T. Li, P. Liu, S. Tao, J. Zhao, D. Han and J. Wang, Effects of oat bran on nutrient digestibility, intestinal microbiota, and inflammatory responses in the hindgut of growing pigs, Int. J. Mol. Sci., 2018, 19, 2407.

31 A. Jahanban-Esfahlan, A. Ostadrahimi, R. JahanbanEsfahlan, L. Roufegarinejad, M. Tabibiazar and R. Amarowicz, Recent developments in the detection of bovine serum albumin, Int. J. Biol. Macromol., 2019, 138, 602-617.

32 K. Eungrasamee, R. Miao, A. Incharoensakdi, P. Lindblad and S. Jantaro, Improved lipid production via fatty acid biosynthesis and free fatty acid recycling in engineered Synechocystis sp. PCC 6803, Biotechnol. Biofuels, 2019, 12, 8.

33 Y. Wang, K. Tang, W. Zhang, W. Guo, Y. Wang, L. Zan and W. Yang, Fatty acid-binding protein 1 increases steer fat deposition by facilitating the synthesis and secretion of triacylglycerol in liver, PLoS One, 2019, 14, e0214144.

34 R. Mesilati-Stahy and N. Argov-Argaman, Changes in lipid droplets morphometric features in mammary epithelial cells upon exposure to non-esterified free fatty acids compared with VLDL, PLoS One, 2018, 13, e0209565.

35 M. Olivares, J. Rodriguez, S. A. Pötgens, A. M. Neyrinck, P. D. Cani, L. B. Bindels and N. M. Delzenne, The janus face of cereals: wheat-derived prebiotics counteract the detrimental effect of gluten on metabolic homeostasis in mice fed a high-fat/high-sucrose diet, Mol. Nutr. Food Res., 2019, 63, 1900632.

36 I. Kimura, J. Miyamoto, R. Ohue-Kitano, K. Watanabe, T. Yamada, M. Onuki, R. Aoki, Y. Isobe, D. Kashihara, D. Inoue, A. Inaba, Y. Takamura, S. Taira, S. Kumaki, M. Watanabe, M. Ito, F. Nakagawa, J. Irie, H. Kakuta, M. Shinohara, K. Iwatsuki, G. Tsujimoto, H. Ohno, M. Arita, H. Itoh and K. Hase, Maternal gut microbiota in pregnancy influences offspring metabolic phenotype in mice, Science, 2020, 367, 1002.

37 E. E. Yalinbas, C. Binay, E. Simsek and M. A. Aksit, The role of umbilical cord blood concentration of IGF-I, IGF-II, leptin, adiponectin, ghrelin, resistin, and visfatin in fetal growth, Am. J. Perinatol., 2019, 36, 600-608.

38 C. Le Bourgot, S. Ferret-Bernard, E. Apper, B. Taminiau, A. Cahu, L. Le Normand, F. Respondek, I. Le HuërouLuron and S. Blat, Perinatal short-chain fructooligosaccharides program intestinal microbiota and 
improve enteroinsular axis function and inflammatory status in high-fat diet-fed adult pigs, FASEB J., 2019, 33, 301-313.

39 L. Zhao, Q. Zhang, W. Ma, F. Tian, H. Shen and M. Zhou, A combination of quercetin and resveratrol reduces obesity in high-fat diet-fed rats by modulation of gut microbiota, Food Funct., 2017, 8, 4644-4656.

40 F. Kong, Y. Hua, B. Zeng, R. Ning, Y. Li and J. Zhao, Gut microbiota signatures of longevity, Curr. Biol., 2016, 26, R832-R833.

41 J. K. Goodrich, J. L. Waters, A. C. Poole, J. L. Sutter, O. Koren, R. Blekhman, M. Beaumont, W. Van Treuren, R. Knight and J. T. Bell, Human genetics shape the gut microbiome, Cell, 2014, 159, 789-799.

42 L. Han, H. Lei, Z. Tian, X. Wang, D. Cheng and C. Wang, The immunomodulatory activity and mechanism of docosahexenoic acid (DHA) on immunosuppressive mice models, Food Funct., 2018, 9, 3254-3263.

43 J. Zou, B. Chassaing, V. Singh, M. Pellizzon, M. Ricci, M. D. Fythe, M. V. Kumar and A. T. Gewirtz, Fibermediated nourishment of gut microbiota protects against diet-induced obesity by restoring IL-22-mediated colonic health, Cell Host Microbe, 2018, 23, 41-53 e44.

44 K. A. Fitzgerald and J. C. Kagan, Toll-like receptors and the control of immunity, Cell, 2020, 180, 1044-1066.

45 R. C. Robertson, A. R. Manges, B. B. Finlay and A. J. Prendergast, The human microbiome and child growth-first 1000 days and beyond, Trends Microbiol., 2019, 27, 131-147.

46 S. Tao, Y. Bai, T. Li, N. Li and J. Wang, Original low birth weight deteriorates the hindgut epithelial barrier function in pigs at the growing stage, FASEB J., 2019, 33, 9897-9912.

47 J. R. Turner, Molecular basis of epithelial barrier regulation: from basic mechanisms to clinical application, Am. J. Pathol., 2006, 169, 1901-1909.

48 B. Ersoy, K. Ozbilgin, E. Kasirga, S. Inan, S. Coskun and I. Tuglu, Effect of growth hormone on small intestinal homeostasis relation to cellular mediators IGF-I and IGFBP-3, World J. Gastroenterol., 2009, 15, 5418-5424.

49 M. H. Ulshen, R. H. Dowling, C. R. Fuller, E. M. Zimmermann and P. K. Lund, Enhanced growth of small bowel in transgenic mice overexpressing bovine growth hormone, Gastroenterology, 1993, 104, 973-980.

50 C. Yue, W. Wang, W. L. Tian, Q. Huang, R. S. Zhao, Y. Z. Zhao, Q. R. Li and J. S. Li, Lipopolysaccharideinduced failure of the gut barrier is site-specific and inhibitable by growth hormone, Inflammation Res., 2013, 62, 407-415.

51 J. Zhang, L. Song, Y. Wang, C. Liu, L. Zhang, S. Zhu, S. Liu and L. Duan, Beneficial effect of butyrate-producing Lachnospiraceae on stress-induced visceral hypersensitivity in rats, J. Gastroenterol. Hepatol., 2019, 34, 1368-1376.
52 L. Peng, Z. R. Li, R. S. Green, I. R. Holzman and J. Lin, Butyrate enhances the intestinal barrier by facilitating tight junction assembly via activation of AMP-activated protein kinase in Caco-2 cell monolayers, J. Nutr., 2009, 139, 1619-1625.

53 M. M. Pusceddu, K. Murray and M. G. Gareau, Targeting the microbiota, from irritable bowel syndrome to mood disorders: focus on probiotics and prebiotics, Curr. Pathobiol. Rep., 2018, 6, 1-13.

54 C. G. Buffie and E. G. Pamer, Microbiota-mediated colonization resistance against intestinal pathogens, Nat. Rev. Immunol., 2013, 13, 790-801.

$55 \mathrm{~J}$. Chen and L. Vitetta, Inflammation-Modulating Effect of butyrate in the prevention of colon cancer by dietary fiber, Clin. Colorectal Cancer, 2018, 17, e541-e544.

56 S. Perego, V. Sansoni, G. Banfi and G. Lombardi, Sodium butyrate has anti-proliferative, pro-differentiating, and immunomodulatory effects in osteosarcoma cells and counteracts the TNFalpha-induced low-grade inflammation, Int. J. Immunopathol. Pharmacol., 2018, 32, 394632017752240.

57 X. Gao, Y. Chen, Z. Chen, Z. Xue, Y. Jia, Q. Guo, Q. Ma, M. Zhang and $\mathrm{H}$. Chen, Identification and antimicrobial activity evaluation of three peptides from laba garlic and the related mechanism, Food Funct., 2019, 10, 4486-4496.

58 T. Tsukahara, K. Watanabe, T. Watanabe, H. Yamagami, M. Sogawa, T. Tanigawa, M. Shiba, K. Tominaga, Y. Fujiwara, K. Maeda, K. Hirakawa and T. Arakawa, Tumor necrosis factor alpha decreases glucagon-like peptide-2 expression by up-regulating G-protein-coupled receptor 120 in Crohn disease, Am. J. Pathol., 2015, 185, 185-196.

59 K. M. Maslowski, A. T. Vieira, A. Ng, J. Kranich, F. Sierro, D. Yu, H. C. Schilter, M. S. Rolph, F. Mackay, D. Artis, R. J. Xavier, M. M. Teixeira and C. R. Mackay, Regulation of inflammatory responses by gut microbiota and chemoattractant receptor GPR43, Nature, 2009, 461, 12821286.

60 E. Chun, S. Lavoie, D. Fonseca-Pereira, S. Bae, M. Michaud, H. R. Hoveyda, G. L. Fraser, C. A. G. Comeau, J. N. Glickman and M. H. Fuller, Metabolite-sensing receptor Ffar2 regulates colonic group 3 innate lymphoid cells and gut immunity, Immunity, 2019, 51, 871-884 e876.

61 F. Rodriguez-Pacheco, C. Gutierrez-Repiso, S. GarciaSerrano, M. A. Alaminos-Castillo, A. Ho-Plagaro, S. Valdes, J. Garcia-Arnes, M. Gonzalo, R. J. Andrade, F. J. MorenoRuiz, A. Rodriguez-Cañete, A. Martinez-Ferriz and E. Garcia-Fuentes, The pro-/anti-inflammatory effects of different fatty acids on visceral adipocytes are partially mediated by GPR120, Eur. J. Nutr., 2016, 56, 1743-1752. 